Review Article

\title{
Long Noncoding RNAs in Yeast Cells and Differentiated Subpopulations of Yeast Colonies and Biofilms
}

\author{
Derek Wilkinson, ${ }^{1}$ Libuše Váchová $\mathbb{D}^{2},{ }^{2}$ Otakar Hlaváček $\left(D,{ }^{2}\right.$ Jana Maršíková, ${ }^{1}$ \\ Gregor D. Gilfillan $\left(\mathbb{D},{ }^{3}\right.$ and Zdena Palková $(\mathbb{1})^{1}$ \\ ${ }^{1}$ Faculty of Science, Charles University, BIOCEV, 25250 Vestec, Czech Republic \\ ${ }^{2}$ Institute of Microbiology of the Czech Academy of Sciences, BIOCEV, 25250 Vestec, Czech Republic \\ ${ }^{3}$ Department Medical Genetics, Oslo University Hospital and University of Oslo, 0450 Oslo, Norway
}

Correspondence should be addressed to Zdena Palková; zdenap@natur.cuni.cz

Received 26 October 2017; Accepted 7 February 2018; Published 25 March 2018

Academic Editor: Paula Ludovico

Copyright ( 2018 Derek Wilkinson et al. This is an open access article distributed under the Creative Commons Attribution License, which permits unrestricted use, distribution, and reproduction in any medium, provided the original work is properly cited.

We summarize current knowledge regarding regulatory functions of long noncoding RNAs (lncRNAs) in yeast, with emphasis on lncRNAs identified recently in yeast colonies and biofilms. Potential regulatory functions of these lncRNAs in differentiated cells of domesticated colonies adapted to plentiful conditions versus yeast colony biofilms are discussed. We show that specific cell types differ in their complements of lncRNA, that this complement changes over time in differentiating upper cells, and that these lncRNAs target diverse functional categories of genes in different cell subpopulations and specific colony types.

\section{Introduction}

Saccharomyces cerevisiae strains used in the brewing industry and in microbiology and genetics laboratories are often grown as planktonic cells in liquid culture, but yeasts also form multicellular communities such as colonies and biofilms, which reflect a more natural lifestyle and are able to cope with different intrinsic and extrinsic stresses [1]. There is growing evidence of cell differentiation, metabolic reprogramming, activation of various stress-defence mechanisms, and other aspects of primitive multicellularity, not only in the complex colony biofilms of nutritionally challenged wild yeast but also in the less structured, smooth colonies of pampered laboratory strains [2-5]. Ammonia signalling, metabolic reprogramming, mitochondrial retrograde signalling, the presence of extracellular matrix, chromosome rearrangement, and many other processes have been described that contribute towards the colony lifestyle, differentiation processes, stress resistance, adaptation, and longevity of multicellular populations [1, 3-7]. However, lncRNA has, until recently, been overlooked as a potential regulator of processes involved in long-term colony development and differentiation, despite the key roles of regulatory ncRNAs in mammalian cell differentiation [8]. The RNAi machinery, which contributes to the production of regulatory ncRNA in many organisms, has been lost in S. cerevisiae [9]. Studies in yeast [10-12] identified large numbers of "cryptic transcripts," "nonannotated transcripts," and "heterogenous unstable RNAs," respectively. These studies established the use of tiling arrays for the identification of yeast long noncoding RNA (lncRNA) and deletion of genes encoding exonucleases, such as RRP6, to stabilise unstable transcripts. Loss of RNAi machinery may have triggered the evolution of a large complement of highly expressed lncRNA in yeast [13]. The detection of several thousand lncRNAs in two studies $[14,15]$ led to an explosion of interest in these poorly understood transcripts.

Here, we present a mini review of yeast lncRNAs and their previously described roles in regulating gene expression under various circumstances. In the second part of the review, we focus in more detail on lncRNAs that we have recently identified in differentiated subpopulations of cells from two distinct types of yeast populations [16, 17], each of which uses unique strategies to cope with stress and ensure 


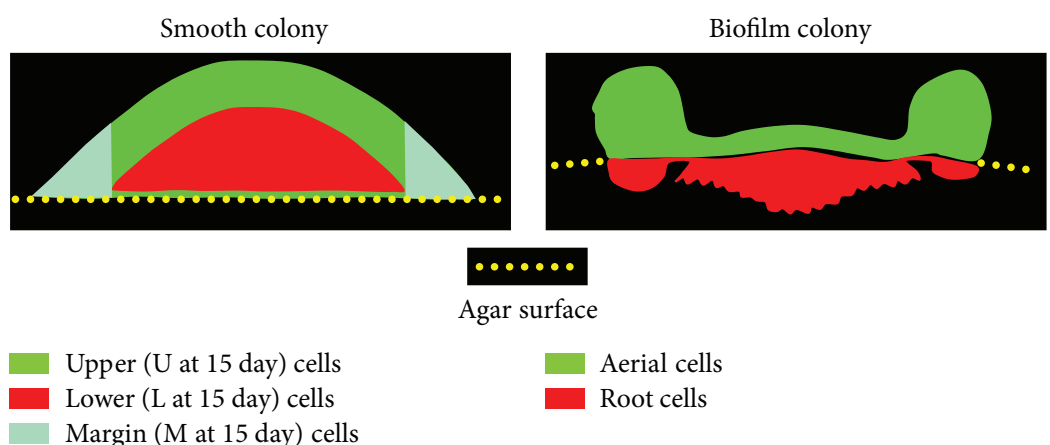

FIGURE 1: Diagram of cell subpopulations isolated from smooth colonies of BY4742 strain (a) and biofilm colonies of BR-F strain (b).

longevity of the population as a whole. These are complex colony biofilms formed by wild strains of Saccharomyces cerevisiae [18] and smooth colonies of S. cerevisiae laboratory strains [19] (Figure 1). We present further analyses of these lncRNAs, particularly in relation to their different types and positions in relation to neighbouring genes. We also discuss potential regulatory activities of lncRNAs in ageing smooth colonies and colony biofilms in light of current knowledge of regulatory functions of lncRNAs in yeast cells.

\section{Important Messages or Random SPAM?}

Transcription of yeast lncRNA occurs largely from bidirectional promoters shared with other loci [10, 14, 20-23]. However, lncRNA accumulation is countered by early termination of unstable antisense transcription, modulation of strand expression via chromatin remodelling, and degradation of lncRNAs [14, 15, 22, 24]. IncRNA/gene expression correlation $[25,26]$ suggests that some lncRNAs are true cellular regulators. Furthermore, there are numerous examples of the stabilisation (or destabilisation) of lncRNA transcript classes under specific conditions, such as meiosis, respiration or sporulation [26-29], carbon source [14, 30], metal abundance [31], and osmotic stress [32]. It was recently shown that the $5^{\prime}-3^{\prime}$ exonuclease $\operatorname{Xrn} 1 \mathrm{p}$ is localised to eisosomes when glucose is scarce but relocalises to the cytoplasm when glucose is present, where it degrades lncRNAs called XUTs and modulates lncRNA regulation of gene expression [33]. Whether this phenomenon constitutes primary regulation or merely "fine-tuning" of gene expression remains to be determined. Nonetheless, it is clear that the study of IncRNA in yeast may uncover important regulatory mechanisms. On the other hand, some lncRNA transcription may simply be a by-product of bidirectional transcription [15].

\section{Classes of lncRNA: Stability and Detection}

The identification of different classes of lncRNAs in yeast has been largely determined by the techniques used in their detection. Microarrays, $3^{\prime}$-long serial analysis of gene expression (SAGE), and RRP6 deletion were used to identify stable unannotated transcripts (SUTs), IncRNAs that are processed in the cytosol similarly to mRNAs, and cryptic unstable transcripts (CUTs) that are sensitive to the RNA decay machinery and degraded by the nuclear exosome and/or the cytoplasmic $5^{\prime}-3^{\prime}$ exonuclease Xrn1p [14, 15, 34]. Other lncRNAs follow this stability-based nomenclature (Table 1), which will also be used in this text. MUTs (meiotic unstable transcripts) are a subset of CUTs that are degraded by Rrp6p RNase (a component of the nuclear exosome complex (NEC)) which accumulate predominantly during meiotic development due to decreased levels of Rrp6p [28]. rsCUTs are expressed during respiration and/or sporulation [28]. XUTs (Xrn1p-sensitive unstable transcripts) are another class of CUTs that are degraded in the cytoplasm. Deletion of XRN1, which encodes a $5^{\prime}-3^{\prime}$ exonuclease, stabilises XUTs [34]. Reducing Nrd1p levels in the nucleus inhibits Nrd1p-dependent lncRNA transcription termination [22], allowing identification of Nrd1-unterminated transcripts (NUTs). Telomeric repeat-containing RNA (TERRA), regulating telomere function, is stabilised in rat 1-1 mutants which are defective in $5^{\prime}-3^{\prime}$ nuclear exonuclease activity [35]. NAM7 (UPF1) encodes an RNA helicase involved in the nonsense-mediated decay (NMD) pathway, and deletion of NAM7 facilitates accumulation of $5^{\prime}$-extended transcripts which, because their accumulation depends on inactivation of cytoplasmic degradation, were termed "cytoplasmically degraded CUTs" (CD-CUTs) [31].

Termination of CUT transcription occurs via a different mechanism from that of mRNAs [36]. The Nrd1p-Nab3p complex, which includes helicase and cap-binding proteins, binds specific motifs that are enriched in CUTs and targets nascent lncRNAs for adenylation and degradation via interactions with the TRAMP complex and nuclear exosome [36-39]. CUTs tend to be degraded in the nucleus and are largely sequestered from the cytoplasm under natural conditions, but other classes of lncRNA are exported from the nucleus and degraded in the cytoplasm [39]. Some lncRNA (e.g., CD-CUTs) may even be imported back into the nucleus, following NMD, where they repress gene expression in trans $[31,39]$. Once lncRNAs are exported from the nucleus, they may be targeted by several decay pathways [40], including (i) deadenylation, decapping, and degradation by the $5^{\prime}-3^{\prime}$ exonuclease Xrn1p; (ii) deadenylation, followed by $3^{\prime}-5^{\prime}$ degradation by the cytoplasmic exosome or via one of the translation-associated pathways; (iii) the nonsense-mediated decay pathway (degrading mRNA 
TABLE 1: Landmarks in the study of yeast lncRNA.

\begin{tabular}{|c|c|c|c|c|}
\hline Discovery & lncRNA class* & Strain manipulation & Technique & Reference \\
\hline Cryptic Pol II transcripts & & $R R P 6$ deletion & Microarray & {$[12]$} \\
\hline Nonannotated transcripts & & Wild type & Tiling array & {$[10]$} \\
\hline CUT termination dependent on Nab3p & & NAB3 mutation & Microarray & [37] \\
\hline Heterogenous unstable RNAs & & $R R P 6$ deletion & Microarray & [11] \\
\hline Telomeric repeat-containing RNAs (TERRAs) & TERRAs & rat1-1 mutants & RT-PCR, northerns & [35] \\
\hline Cryptic unstable transcripts (CUTs) & CUTs & RRP6 \& TRF4 deletion & 3'-long SAGE & {$[14]$} \\
\hline Stable unannotated transcripts (SUTs) \& CUTs & SUTs, CUTs & RRP6 deletion & Microarray & {$[15]$} \\
\hline PHO84 antisense lncRNA can repress in trans & & Ectopic PHO84 expression & qPCR, northerns & {$[59]$} \\
\hline PWR1/ICR1 lncRNAs and FLO11 expression & & $\Delta P_{F L O 11}$, cit6, and sfl 1 & Northern blot & {$[41]$} \\
\hline Condition-dependent antisense transcripts & & Stationary phase, etc. & Stranded RNA-seq & {$[26]$} \\
\hline $\begin{array}{l}\text { Meiotic unannotated transcripts (MUTs), } \\
\text { respiration/sporulation unannotated } \\
\text { transcripts (rsCUTs) }\end{array}$ & MUTs, rsCUTs & Meiotic a/ $\alpha$ diploids & Tiling array & {$[28]$} \\
\hline Xrn1-sensitive unstable transcripts (XUTs) & XUTs & $X R N 1$ deletion & RNA-seq & {$[34]$} \\
\hline RME2 lncRNA regulates IME2 expression & & $R M E 2$ promoter deletion & RT-PCR & [27] \\
\hline CUT repression of metal homeostasis genes & CD-CUTs & NMD/CD-CUT mutants & Northern blot & [31] \\
\hline$I M E 1$ and $I M E 4$ expression regulated by $\operatorname{lncRNA}$ & & set2, set3, haploid/diploid & ChIP, northerns & [29] \\
\hline lncRNA/gene pairs, coregulated in colonies & & GFP-tagged ATO1 & Microarrays & {$[66]$} \\
\hline Nrd1-unterminated transcripts (NUTs) & NUTs & FRB-tagged NRD1 & $4 \mathrm{tU}$-seq & {$[22]$} \\
\hline Stress/Hoglp-regulated lncRNA transcription & & HOG1 deletion & Tiling arrays & {$[32]$} \\
\hline
\end{tabular}

*Italics: lncRNA classes discovered; bold: classes discussed here in relation to smooth and biofilm colonies.

containing, e.g., spurious stop codons); and (iv) no-go decay (NGD: degrading mRNA with stalled elongation). Wery et al. [40] review the high degree of overlap between IncRNA classes (e.g., between XUTs and both SUTs and CUTs) and suggest that many XUTs are merely SUTs that have been extended at the $3^{\prime}$ end and that these extensions target XUTs for NMD.

\section{Regulation of Gene Expression by Annotated lncRNAs}

lncRNAs differ in terms of distance from coding regions and orientation with regard to coding genes (Figure 2(a)). Antisense transcripts are transcribed from overlapping loci, located on the opposite strand to sense loci [26] (antisenseoverlapping). lncRNA/gene pairs on opposite strands may be transcribed from nearby start points but in opposite directions (antisense-divergent orientation), or their transcription may converge on a common end point (antisense-convergent orientation). Alternatively, the lncRNA locus may occur on the same strand as an upstream or downstream locus (tandem sense orientation) [14].

The functions of most yeast lncRNAs identified so far are unknown, but there are some exceptions, including regulation of GAL1, PHO5, and PHO84 expression [23], in which the lncRNA has been assigned a role in regulating the expression of a related gene. In some of these examples, the regulatory function is attributed to the transcription process itself and not to the presence of the lncRNA transcript [39]. For example, lncRNA transcription could be involved in changes in chromatin structure that subsequently influence the binding of transcription factors to promoter regions of the related gene, as was shown for the negative regulation of IME1 [29] and modulation of expression of FLO11 [41]. Other known mechanisms of lncRNA function include transcriptional interference in which antisense lncRNA can block/decrease transcription of sense-strand mRNA, for example, in the case of IME4 mRNA transcription [29]. Many lncRNAs are regulated by the same promoter as a divergent gene on the opposite strand [42], and the lncRNA negatively regulates the expression of an antisense-overlapping gene that lies upstream of the antisense-divergent gene.

Expression of the FLO11 gene, involved in many processes including colony biofilm formation, is modulated by the expression of a tandem upstream sense locus, ICR1. ICR 1 expression is in turn regulated by an antisenseoverlapping locus, PWR1 [41] in a "toggle"-like manner, dependent on two transcription factors: Sfl1p activates ICR1 expression, inhibiting FLO11 expression, while Flo8p upregulates $P W R 1$ expression, inhibiting ICR1 expression and promoting FLO11 expression [41]. Rpd3p-mediated chromatin modification may block Sfl1p binding, countering ICR1-mediated inhibition of FLO11 expression. Bumgarner et al. [43] revealed the existence of three expression states within a clonal cell population in which the FLO11 promoter is (i) silenced, (ii) nonsilenced but lacking transcription factors, and (iii) nonsilenced and bound by transcription factors leading to FLO11 expression that is absent, low, and high, respectively. FLO11 expression correlated with PWR1 expression and anticorrelated with ICR1 expression and the physical act of ICR 1 transcription 


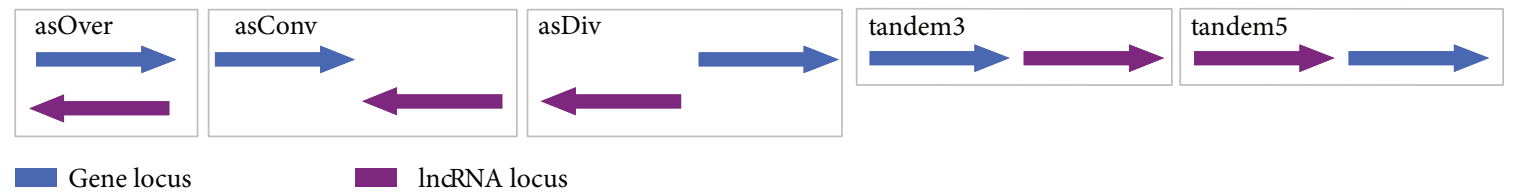

(a)

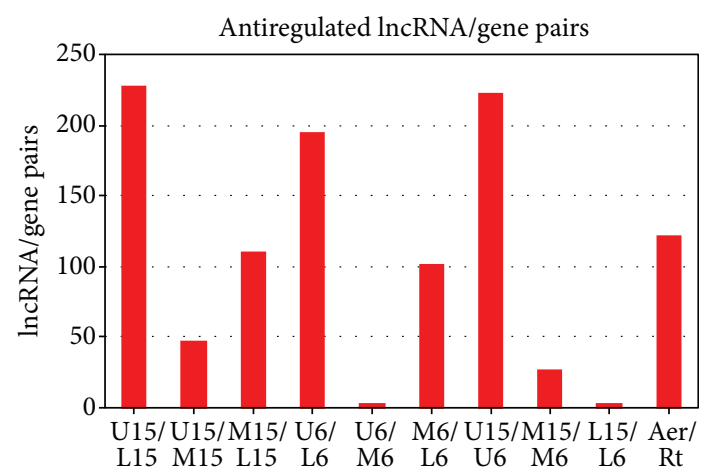

(b)

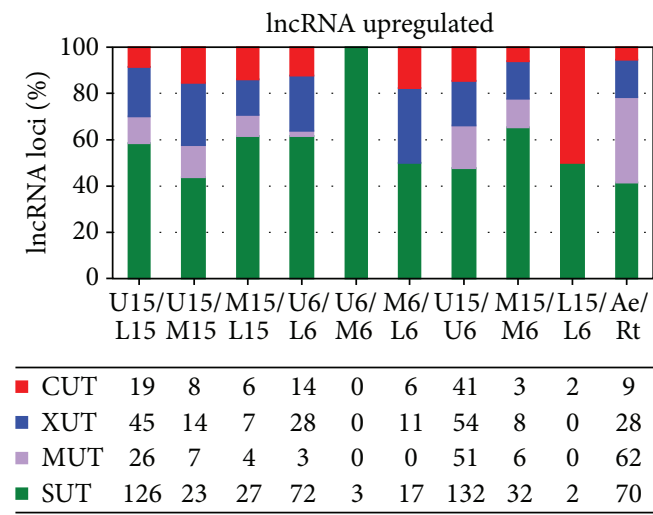

(d)

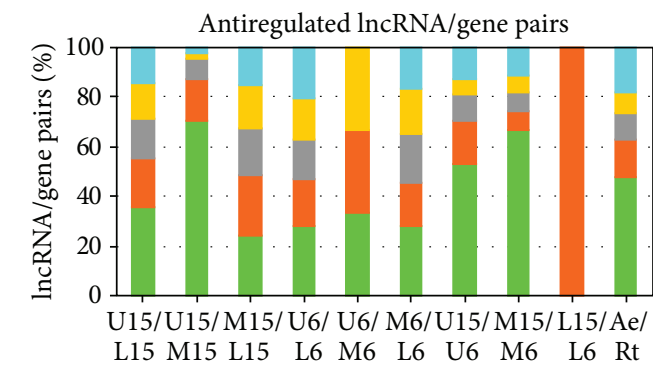

\begin{tabular}{lllllllllll}
\hline$\square$ tandem5 & 33 & 1 & 17 & 40 & 0 & 17 & 28 & 3 & 0 & 22 \\
$\square$ tandem3 & 33 & 1 & 19 & 32 & 1 & 18 & 14 & 2 & 0 & 10 \\
$\square$ asDiv & 36 & 4 & 21 & 32 & 0 & 20 & 23 & 2 & 0 & 13 \\
$\square$ asConv & 44 & 8 & 26 & 36 & 1 & 18 & 40 & 2 & 2 & 18 \\
$\square$ asOver & 81 & 33 & 27 & 55 & 1 & 28 & 117 & 18 & 0 & 58 \\
\hline
\end{tabular}

(f)

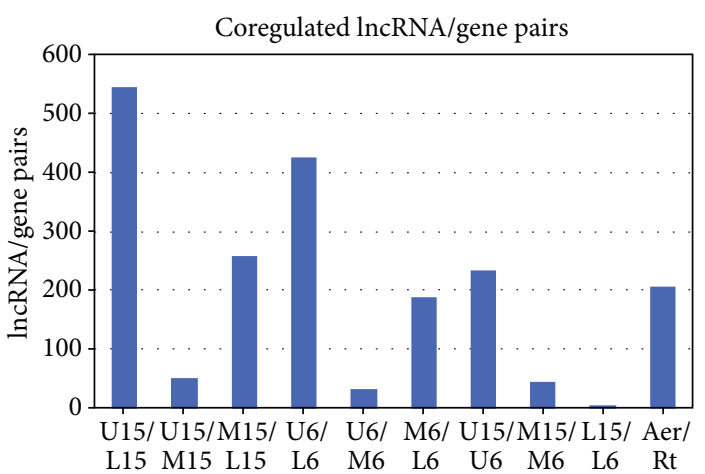

(c)

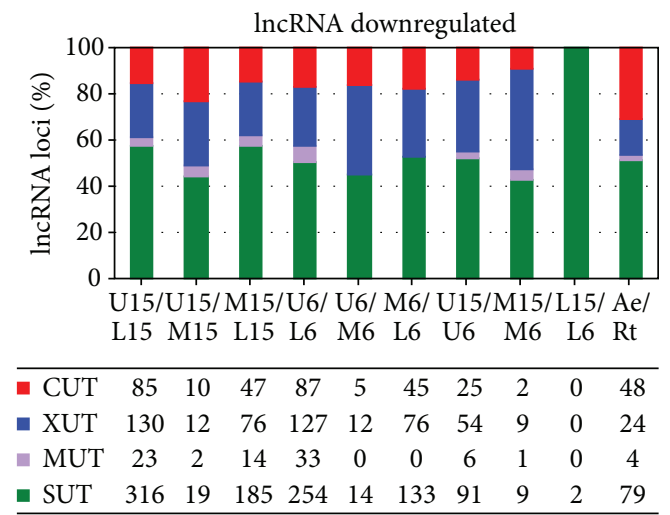

(e)

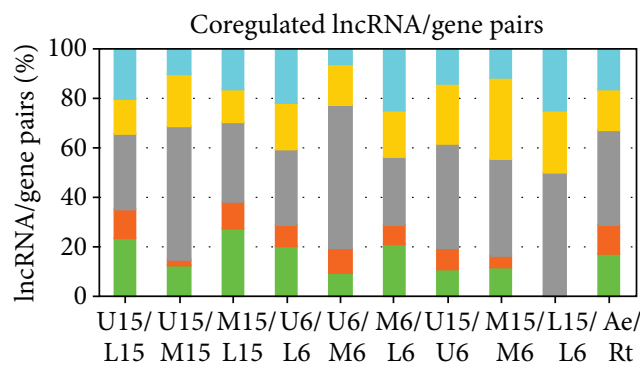

\begin{tabular}{lcccccccccc}
\hline$\square$ tandem5 & 109 & 5 & 41 & 94 & 2 & 47 & 32 & 5 & 1 & 33 \\
$\square$ tandem 3 & 78 & 10 & 34 & 78 & 5 & 35 & 56 & 14 & 1 & 33 \\
$\square$ asDiv & 166 & 26 & 82 & 129 & 18 & 51 & 99 & 17 & 2 & 79 \\
$\square$ asConv & 61 & 1 & 28 & 37 & 3 & 15 & 19 & 2 & 0 & 23 \\
asOver & 129 & 6 & 71 & 85 & 3 & 39 & 26 & 5 & 0 & 35 \\
\hline
\end{tabular}

(g)

FIGURE 2: lncRNAs detected in subpopulations of aged yeast colony cells. DE gene and DE lncRNA loci were identified that were located within $1.5 \mathrm{kB}$ of one another in 5 different orientations (a). The numbers of antiregulated (b) and coregulated (c) lncRNA/gene pairs were compared for the different expression comparisons detailed at the bottom of the figure. Percentages (bars) and numbers (boxes underneath) of different classes of lncRNA upregulated (d) or downregulated (e) in each sample as well as the percentages (bars) and numbers (boxes) of antiregulated (f) and coregulated (g) lncRNA/gene pairs are given for each comparison. Abbreviations: asOver: antisense-overlapping; asConv: antisense-convergent; asDiv: antisense-divergent; tandem3: tandem to (sense strand) and downstream of gene; tandem5: tandem to (sense strand) and upstream of gene; SUT: stable unannotated transcript; MUT: meiotic unstable transcript; XUT: Xrn1-dependent unstable transcript; CUT: cryptic unannotated transcript. 
displaced transcription factors from the FLO11 promoter, effectively "resetting" the promoter. Cells with fewer than 5 fluorescent Flo11p dots lacked structured colony morphology, diploid pseudohyphae formation, and haploid adhesion. Since Flo11p drives biofilm formation and biofilm development is a key fungal virulence factor in pathogenic fungi such as Candida albicans [44-46], identifying regulators of FLO11 expression could help to counter the high drug resistance of fungal biofilms or uncover drug targets for treatment of potentially lethal fungemias.

In haploid cells, Rmelp binds upstream of the key meiotic regulator $I M E 1$, causing the tandem sense locus IRT1 to express a transcript that recruits histone remodellers that produce a repressive chromatin structure over the IME1 promoter [29]. In MATa/ $\alpha$ diploids, the a1- $\alpha 2$ repression complex blocks RME1 expression, relieving inhibition of meiosis. Another major regulator of meiosis is IME4, encoding a protein that methylates many key sporulation mRNAs $[27,29] . R M E 2$, an antisense IncRNA locus overlapping the whole IME4 ORF, inhibits IME4 expression in haploid cells. However in diploid cells, the a1- $\alpha 2$ complex binds to the $R M E 2$ promoter, inhibiting lncRNA expression and relieving the block on IME4 expression [29]. In another example of gene expression regulation via chromatin remodelling, the osmotic stress-induced MAPK, Hoglp binds the $3^{\prime}$ end of CDC28 (a master regulator of mitosis and meiosis), promoting antisense lncRNA expression that triggers looping of the CDC28 gene. This looping allows Hog1p to "jump" across the narrow neck of the loop from the $3^{\prime}$ to the $5^{\prime}$ end, where it induces RSC-dependent chromatin remodelling, leading to CDC28 gene expression [32]. These examples demonstrate how lncRNA regulatory mechanisms can be switched on or off in specific cell subpopulations or in the presence of stress [27, 32].

In some cases, transcription of a lncRNA directly hinders gene expression from a neighbouring target gene [39]. When serine is plentiful, expression from a $5^{\prime}$ tandem sense lncRNA locus, SRG1, inhibits expression of the serine biosynthesis gene SER3 by increasing promoter nucleosome density, which prevents transcription factors from accessing the promoter [47]. Martens et al. [48] showed that serine availability activates binding of the transcription factor Cha4p to the SRG1 promoter and recruitment the Swi/Snf and SAGA complexes, leading to activation of SRG1 and thus repression of SER3. Such "transcriptional interference" is a common mechanism by which the expression of one locus is attenuated by that of a second, converging locus [49], not only between coding and noncoding loci but also between converging coding loci.

A GAL10 antisense-overlapping lncRNA (which also sense-overlaps GAL1) has been shown to recruit methyltransferase and deacetylase complexes which silence both GAL10 and GAL1 [39]. Pinskaya et al. [50] showed that glucose/Reb1-dependent transcription of the antisense transcript GAL1 $1_{\text {uncut }}$ promotes Set1-dependent H3K4 di/trimethylation and facilitates recruitment of the RPD3S histone deacetylase, repressing GAL1. A similar mechanism blocks transcription from a "hidden" promoter within SUC2, suggesting that $\mathrm{H} 3 \mathrm{~K} 4 \mathrm{di} /$ trimethylation might represent a widespread mechanism for maintaining promoter fidelity. lncRNAs are decapped and degraded in a DCP1-, DCP2-, XRN1-, and RAT1-dependent manner [51] to facilitate galactose-induced GAL expression. Lenstra et al. [52] showed that IncRNA expression has two modes: spurious and functional. When GAL expression is induced, GAL10 expression is independent of spurious lncRNA expression, but when not induced, tight repression of GAL10 is dependent upon functional IncRNA expression. Cloutier et al. and Beck et al. [53-56] demonstrated that the glucose-dependent DEAD box RNA helicase negatively regulates formation of DNA/RNA hybrid R-loops and that a change of carbon source from glucose to galactose promotes export of Dbp2p to the cytoplasm and IncRNAdependent R-loop formation and displacement of the Cyc8Tup1p corepressor from the promoter and derepression of GAL genes. Thus, relieving the block on R-loop formation may be a general mechanism for rapidly derepressing key genes and adapting to changing environmental conditions [55]. Zacharioudakis and Tzamarias [57] further showed that when galactose concentration is high, Gallp enters a positive feedback loop with Gal4p and the GAL genes are turned on, independently of lncRNA. However, when galactose levels are low, the lncRNA is able to randomly block transition to the on state, delaying the switch to alternative carbon source in a percentage of cells and facilitating metabolic flexibility.

Although most cases of lncRNA regulation identified in budding yeast appear to be cis-acting, some examples of trans-acting regulators have been described [34, 58, 59]. Reference [34] identifies Xrn1p-sensitive antisense transcripts, some with apparent regulatory roles, and suggests a mechanism whereby XUTs interact with a protein complex to silence target genes and that this activity is promoted by histone $\mathrm{H} 3 \mathrm{~K} 4$ mono/di-methylation but opposed by histone $\mathrm{H} 3 \mathrm{~K} 4$ trimethylation. Large numbers of different XUTs are exported from the nucleus and may be degraded by translation-coupled NMD [40], so they may also have posttranscriptional regulatory roles [34]. Ty1 retrotransposon expression is regulated in trans by an antisense CUT [58] which interacts with Ty1, promotes histone deacetylation and Set1-dependent methylation, and effects chromatin silencing. It has been shown [59] that PHO84 antisense lncRNA is able to act in trans to silence transcription of a second copy of PHO84 elsewhere in the genome. Silencing of the second PHO84 copy depends upon a region of homology with the upstream activating sequence and possibly also recruits a silencing complex to the promoter.

A IncRNA overlaps the PHO5 gene on the antisense strand, and transcription of the lncRNA across the gene promoter plays a role in the activation (not repression) of PHO5 [60] by increasing the efficiency of histone removal, facilitating access of the polymerase to the TATA box. Bunina et al. [61] showed that starvation-/sporulationinduced expression of the antisense transcript is dependent upon repetitive regions in the $3^{\prime}$ UTR and has no effect upon promoter activity but does promote the expression of a long mRNA isoform with enhanced stability. 
Chia et al. [62] showed that transcription of an upstream IncRNA, NDC80 luti, represses NDC80 expression during meiotic prophase by driving Set1-dependent H3K4Me2 and Set2-dependent $\mathrm{H} 3 \mathrm{~K} 36 \mathrm{Me} 3$ at the NDC80 promoter, leading to recruitment of the Set3C and Rpd3S histone deacetylases. The pervasiveness of lncRNA regulation of gene expression in different cell types and under differing conditions has been demonstrated in a number of studies. Kim et al. [63] showed that the promoters of a high percentage of Set2-repressed genes are overlapped by antisense or upstream tandem lncRNA that promote H3K36Me3 and Rpd3S-dependent deacetylation within the promoter region and that many of these genes are regulated by carbon source. A similar mechanism involving Set3C-dependent H3K4me2 was identified previously [30]. McDaniel et al. [64] showed that deleting SET2 affects the expression of genes involved in stress responses because lack of $\mathrm{H} 3 \mathrm{~K} 36 \mathrm{Me}$ permits the inappropriate transcription of antisense lncRNA that interferes with gene transcription.

Kwapisz et al. [65] identified CUTs and XUTs, generated from subtelomeric regions (subTERRA) with roles in telomeric silencing and prevention of clustering, respectively, via the formation of DNA-RNA hybrids and by protein scaffolding.

\section{Long ncRNAs May Contribute to Gene Regulation within Differentiated Cell Subpopulations of Colonies and Colony Biofilms}

Traven et al. [66] provided a first glimpse into the presence of, and potential regulation of genes by, $\operatorname{lncRNAs}$ within yeast colonies of laboratory strain BY4741, grown on complete glucose medium and differentiated into two subpopulations of cells on the "outside" and "inside" of the colonies. In this study, transcriptomic differences were identified by microarrays and included 12 SUTs and CUTs on the outside and 53 on the inside of the colonies. In addition, several lncRNA/gene pairs with positively correlating expression were identified that represent possible examples of gene regulation by $\operatorname{lncRNA}$, including the ammonium permease gene $M E P 2$.

We performed further studies using the more sensitive RNA sequencing (RNA-seq) technique. RNA-seq provided a detailed transcriptomic view of six cell subpopulations present in smooth BY4742 colonies grown on complete respiratory medium [17]: cells from upper, margin, and lower parts of colonies in two developmental phases (late acidic 6-day-old and alkali-phase 15-day-old). In parallel experiments, two subpopulations (a surface "aerial" cell subpopulation and a subpopulation of invasive "root" cells growing within the agar) from structured colony biofilms grown on the same medium were also studied [16] (Figure 1). Every gene located within $1.5 \mathrm{kB}$ of (or antisense-overlapping) each lncRNA was identified to produce a list of lncRNA/gene pairs in any of the 5 different orientations (Figure 2(a)) considered. Previous expression profiling of subpopulations of smooth colonies and colony biofilms identified some metabolic similarities but even more differences $[16,17]$. Whereas some of the expression differences in individual genes may be caused by the fact that laboratory and wild strains forming smooth colonies and colony biofilms, respectively, are not isogenic, most of the differences are in agreement with the different lifestyles of yeast populations in smooth colonies (formed by either laboratory strains or domesticated wild strains) versus colony biofilms $[1,5]$. Here, we therefore also compared types of identified lncRNAs as well as lncRNA/ gene pairs in smooth colonies and colony biofilms, to see whether any potential lncRNA-related similarities exist among subpopulations of these structures.

5.1. Antiregulation of IncRNA/Gene Pairs Is Highest when Comparing Dissimilar Cell Types. Cells localised to the upper and marginal regions of smooth colonies (Figure 1) are somewhat similar in gene expression, protein production, and so on and are more different from cells localised to the lower regions of central colony areas [17]. lncRNA/gene antiregulation (where gene and lncRNA are antagonistically differentially expressed in the two subpopulations) and coregulation (agonistically differentially expressed) were highest when upper (U) and lower (L) cells were compared and lowest when upper and margin $(\mathrm{M})$ cells were compared (Figures 2(b) and 2(c)). Comparison of aerial and root cells of colony biofilms revealed numbers of antiregulated and coregulated pairs, closest to the numbers identified in marginal/lower cell comparisons in smooth colonies (Figures 2(b) and 2(c)). The numbers of mapped reads were similar for biofilm and smooth colonies (average of 17.1 and 15.4 million reads, resp.); the percentages of mapped reads mapping to lncRNA were 23 and 25\%, respectively; and the same read counting and differential expression analysis packages were used in both analyses.

Increased antiregulation and coregulation in the $\mathrm{U} / \mathrm{L}$ and $\mathrm{M} / \mathrm{L}$ comparisons, compared with U/M (in both 6- and 15-day-old colonies), is consistent with observed U/M cell similarities (metabolic, gene expression, and other) and differences of both from L cells [17]. High U6/U15 antiand coregulation agrees with the finding that temporal gene expression changes are most prominent in upper cells of developing colonies [17]. Surprisingly, approximately twice as many antiregulated/coregulated pairs were observed when comparing upper versus lower cells (in smooth colonies) than in root versus aerial parts of biofilm colonies. However, aerial and root parts of colony biofilms are not homogenous and contain small subpopulations of cells with features typical of their counterparts [16]. This fact may dilute the observed aerial-root cell differences. Furthermore, aerialroot cells were separated from younger colony biofilms (3-day-old) than the cells of smooth colonies (6- and 15day-old), in which upper cells gradually acquire unique metabolic features and gain specific physiology important for longevity $[19,67]$. In contrast, only moderate expression changes occur during this time period in slowly growing marginal and lower cells [17]. Accordingly, expression differences between margin and lower cells are more comparable at different developmental time points. However, the aerial versus root and margin versus lower cell comparisons are 
similar only in terms of the numbers of co- and antiregulated lncRNA/gene pairs, which may reflect merely the level of similarity/dissimilarity between the respective cell types. As shown below, different lncRNA/gene pairs were identified in smooth colonies and colony biofilms.

5.2. Cell-Type-Specific Expression of IncRNA Classes. Numbers of differentially expressed lncRNAs differ significantly when comparing the various cell subpopulations (Figures 2(d) and 2(e)). The terms upregulation/upregulate (or downregulation/downregulate) are relative, so the observations could be caused by activation (or repression) of transcription in the first subpopulation or by repression (or activation) in the second. Lower cells were found to upregulate the highest number of lncRNAs of all monitored cell types, as shown in Figures 2(d) and 2(e). Margin cells upregulate 10 times as many lncRNA loci as upper cells at 6 days, whereas no difference was observed at 15 days, which is consistent with the finding that the number of upregulated lncRNAs in upper cells increases over time. No differences in the total number of up-/downregulated lncRNAs were observed between aerial and root cells of colony biofilms.

SUTs were the most common lncRNAs in smooth colonies ( $>50 \%$ of both upregulated and downregulated lncRNAs) as well as in colony biofilms (40\% of upregulated and $>50 \%$ of downregulated lncRNAs). Differentially expressed SUTs were similarly distributed between upregulated and downregulated lncRNA categories across most comparisons (Figures 2(d) and 2(e)). CUTs form $\sim 15 \%$ of up-/downregulated genes in most comparisons with no significant difference in distribution in smooth colonies. However, significant differences were detected in colony biofilms, where $5.3 \mathrm{x}$ as many CUTs were upregulated in roots (forming $30 \%$ of all upregulated lncRNAs in roots) as in aerial cells.

The clearest difference in up- and downregulated unstable lncRNAs between smooth colonies and colony biofilms was based on MUTs, forming $~ 6.5 \%$ and $20 \%$ of up-/down regulated lncRNAs, respectively. More MUTs are up- or downregulated in 15-day-old smooth colonies than in 6-day-old colonies, in which MUTs were only identified when comparing $\mathrm{L}$ and $\mathrm{U}$ cells (10x as many MUTs were upregulated in L cells), indicating increased MUT expression during smooth colony ageing. The largest group of upregulated MUTs occurred in aerial cells of colony biofilms, $>14 \mathrm{x}$ more than the number of upregulated MUTs in roots. In summary, expression of MUTs is increased in upper (and partially in margin) parts of smooth colonies during ageing, whereas MUTs are already highly expressed in aerial cells of much younger colony biofilms.

Increased MUT expression in aerial cells of biofilm colonies is consistent with the upregulation of a large group of meiotic genes in aerial cells [16]. MUTs accumulate predominantly during meiotic development, possibly due to decreased levels of Rrp6p RNase (a component of NEC), which can degrade MUTs [28] and CUTs [14, 15]. Accordingly, RRP6 expression is $>2$-fold upregulated in root cells than in aerial cells. In smooth colonies, RRP6 expression (which can affect CUT stability) is only moderately upregulated (1.3- to 1.4-fold) in $\mathrm{L}$ relative to $\mathrm{U}$ or $\mathrm{M}$ cells. However, CUT degradation is also dependent upon Nab3p, Nrd1p, and the TRAMP complex (mainly the TRAMP4 complex, which includes Pap2p (Trf4p) [68]). The apparent upregulation of CUT expression in lower cells and MUT expression in upper cells may be due to differential expression of the TRAMP4 and TRAMP5 complexes (which target both shared and unshared transcripts [69]) and of the $3^{\prime}-5^{\prime}$ exosome components Rrp6p and Dis3p.

5.3. Orientation of IncRNA/Gene Pairs Differs in Antiregulated and Coregulated Pairs. IncRNA/gene pairs were classified according to their mutual position (Figure 2(a)) and expressional relationship (antiregulated, Figure 2(f), and coregulated, Figure 2(g)). The total number of coregulated versus antiregulated lncRNA/gene pairs was slightly higher in both smooth colonies $(>1.8 \mathrm{x})$ and colony biofilms $(>1.7 x)$, but more prominent differences were observed between specific cell types and among different lncRNA/gene position categories. Coregulated lncRNA/gene pairs were overrepresented as compared with antiregulated pairs in U15/L15 (>2.3x), M15/L15 (>2.3x), U6/L6 (>2.1x), and U6/M6 (>10x) cell comparisons.

Antisense-overlapping (asOver) lncRNAs were the most common category of antiregulated lncRNA/gene pairs both in smooth colonies (>38\%) and in colony biofilms $(>47 \%)$, whereas antisense-divergent (asDiv) lncRNAs were the most prominent category in coregulated lncRNA/gene pairs ( $>33 \%$ in smooth colonies and $>38 \%$ in colony biofilms). Enrichment of antisense-divergent loci among coregulated and antisense-overlapping loci among antiregulated lncRNA/gene pairs is consistent with previous reports of a positive correlation between the expression of antisense-divergent loci (gene and lncRNA), possibly because of increased bidirectional transcription from a common nucleosome-depleted region [14] and of interference by antisense-overlapping lncRNA in gene expression and thus negative regulation [26]. The distribution of asOver and asDiv lncRNA/gene pairs among different cell comparisons was relatively equal, with the exception of U15/M15 (1.82x more asOver antiregulated pairs than average and $1.65 \mathrm{x}$ more asDiv coregulated pairs than average), U6/M6 (1.74x more asDiv coregulated pairs than average), and M15/M6 (1.73x more asDiv coregulated pairs than average) comparisons.

5.4. Different Functional Groups of Genes May Be Negatively Regulated by IncRNA in Smooth and Biofilm Colonies. The numbers of potentially negatively (in antiregulated lncRNA/gene pairs) and positively (in coregulated lncRNA/ gene pairs) regulated genes in different functional annotation groups were considered. Antiregulated/coregulated lncRNA/ gene pairs were annotated with functional categories using information in SGD (http://www.yeastgenome.org/, [70]) and the literature. Datasets of differentially expressed (DE) genes were then compared using Intervene's UpSet module [71], which visualizes the intersection of multiple data sets in UpSet plots (Figure 3). No common antiregulated and only 6 common coregulated lncRNA/gene pairs were 


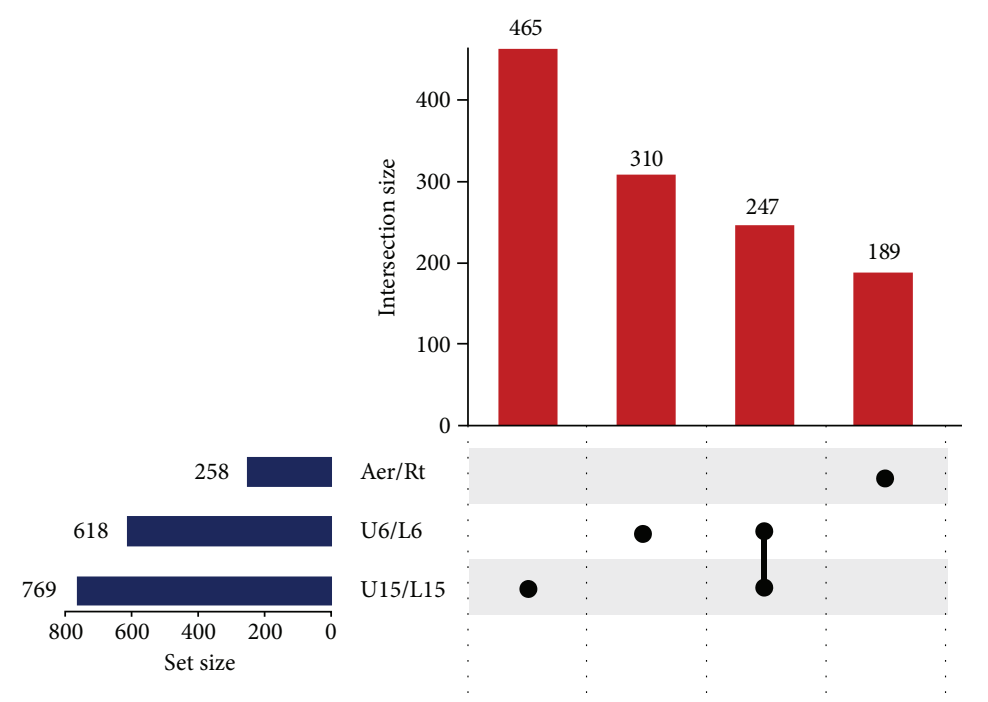

\begin{tabular}{|c|c|c|c|c|c|c|c|c|}
\hline Functional categories & A & $\mathrm{C}$ & A & $\mathrm{C}$ & A & $\mathrm{C}$ & A & $\mathrm{C}$ \\
\hline Amino acid/purine-pyrimidine metabolism & 6 & 10 & 3 & 3 & 1 & 4 & 3 & 9 \\
\hline Carbohydrate metabolism & 3 & 9 & 0 & 5 & 1 & 12 & 3 & 5 \\
\hline Lipid metabolism & 4 & 8 & 2 & 5 & 3 & 4 & 1 & 2 \\
\hline Vitamin/coenzyme metabolism & 4 & 2 & 4 & 1 & 4 & 4 & 1 & 2 \\
\hline Respiration/ATP synthesis & 4 & 6 & 5 & 1 & 0 & 2 & 2 & 0 \\
\hline Metabolism, other & 0 & 2 & 6 & 2 & 0 & 4 & 2 & 0 \\
\hline Transport-amino acids/purine-pyrimidine & 0 & 4 & 0 & 3 & 0 & 0 & 4 & 3 \\
\hline Transport-sugars/carbohydrate & 0 & 3 & 0 & 1 & 0 & 1 & 1 & 5 \\
\hline Ion transport & 6 & 9 & 6 & 7 & 1 & 6 & 0 & 5 \\
\hline Other transport & 5 & 10 & 2 & 6 & 2 & 1 & 3 & 6 \\
\hline Cell wall assembly, maintenance, integrity & 7 & 10 & 2 & 6 & 1 & 10 & 0 & 0 \\
\hline Endocytosis/exocytosis & 4 & 4 & 2 & 1 & 0 & 1 & 1 & 1 \\
\hline Protein trafficking & 3 & 23 & 5 & 10 & 3 & 11 & 0 & 4 \\
\hline Protein maturation/complex assembly & 4 & 7 & 6 & 4 & 0 & 10 & 0 & 1 \\
\hline Organelle assembly/function & 0 & 3 & 3 & 2 & 0 & 0 & 0 & 0 \\
\hline Stress response-DNA damage & 4 & 6 & 1 & 3 & 1 & 1 & 0 & 1 \\
\hline Stress response-oxidative & 3 & 3 & 2 & 4 & 0 & 4 & 0 & 0 \\
\hline Stress response-other & 0 & 3 & 2 & 3 & 0 & 3 & 0 & 2 \\
\hline Cell cycle & 9 & 12 & 8 & 5 & 2 & 6 & 3 & 1 \\
\hline Meiosis/sporulation & 12 & 10 & 2 & 4 & 0 & 9 & 7 & 9 \\
\hline Actin, tubulin, myosin, dynein & 0 & 1 & 0 & 0 & 0 & 0 & 1 & 2 \\
\hline Ribosome biogenesis & 5 & 4 & 5 & 4 & 3 & 4 & 2 & 10 \\
\hline Ribosome subunit & 6 & 9 & 6 & 2 & 8 & 4 & 3 & 7 \\
\hline General transcription & 0 & 4 & 2 & 0 & 1 & 0 & 2 & 1 \\
\hline Translation & 9 & 13 & 9 & 9 & 3 & 5 & 7 & 7 \\
\hline Proteasome/ubiquitin/ubiquitin-like & 2 & 12 & 0 & 4 & 1 & 9 & 3 & 4 \\
\hline Protein degradation, other & 1 & 5 & 1 & 3 & 0 & 7 & 0 & 1 \\
\hline Regulation, transcription & 6 & 13 & 4 & 6 & 1 & 10 & 1 & 4 \\
\hline Regulation, signalling & 16 & 18 & 10 & 15 & 4 & 14 & 2 & 5 \\
\hline Regulation, other & 2 & 6 & 3 & 5 & 4 & 5 & 2 & 0 \\
\hline Retrotransposon & 2 & 2 & 2 & 1 & 0 & 3 & 0 & 0 \\
\hline Other & 5 & 4 & 1 & 2 & 1 & 3 & 0 & 0 \\
\hline Unknown/putative & 19 & 56 & 21 & 47 & 2 & 36 & 10 & 22 \\
\hline Dubious ORF & 6 & 17 & 1 & 10 & 2 & 5 & 3 & 3 \\
\hline
\end{tabular}

FIGURE 3: UpSet plot of datasets of coregulated and antiregulated genes in the upper and lower parts of biofilm colonies (Aer/Rt) and of 6-dayold (U6/L6) and 15-day-old (U15/L15) smooth colonies. Coregulated and antiregulated gene/lncRNA pairs in three cell comparisons: aerial versus roots of biofilm colonies, upper cells versus lower cells of 6-day-old smooth colonies, and upper cells versus lower cells of 15-day-old smooth colonies $[16,17]$. Horizontal blue bar chart indicates numbers of genes, co-/antiregulated (with lncRNA) in each individual comparison. The black dots and lines (intersect "connectors") above the heat map indicate comparisons in which the given number of genes (vertical red bar chart) were co-/antiregulated. Only major intersections are shown. Heat map of genes assigned to functional categories and clustered according to functional category (FC) and co-/antiregulated in different sample comparisons (lower part). Number in heat map cell = number of genes from FC, coregulated or antiregulated with lncRNA in a sample comparison. The higher the number of co-/antiregulated genes, the more intense the colour. A: antiregulated; C: coregulated. 
identified in U15 versus L15, U6 versus L6, and aerial versus root cell comparisons, and these include the genes KSP1, PRC1, YNL200C, LDS2, RRT8, and NCR1, encoding a serine/threonine phosphatase with a putative role in TOR signalling, a vacuolar carboxypeptidase Y, a NADHX epimerase, 2 paralogous spore wall assembly proteins, and a vacuolar membrane protein involved in sphingolipid metabolism, respectively. Over $14 \%$ of antiregulated lncRNA/gene pairs in upper versus lower cells are shared between 6-day- and 15 -day-old smooth colonies, but only $1 \%$ is shared by smooth colonies and colony biofilms (6- or 15-day-old). There are major morphological, expression, and metabolic differences between the two colony types [5, 18, 19], the biofilm colony strain BR-F is diploid while the smooth strain BY4742 is haploid, and signalling and coding RNA expression differences between aerial and root cells may outweigh differences in gene expression regulation by lncRNA.

465 lncRNA/gene pairs were coregulated (308) or antiregulated (157) exclusively in U15 versus L15 cells (Figure 3), including antiregulated genes with roles in regulation/signalling, meiosis/sporulation, cell cycle, translation, and cell wall assembly/maintenance/integrity (Figure 3). 310 pairs were coregulated (184) or antiregulated (126) exclusively in U6 versus L6 cells, including antiregulated genes with roles in regulation/signalling, translation, cell cycle, and ribosomal biogenesis as well as those encoding ribosome subunits (Figure 3). These data suggest that development of upper cells may be partially dependent upon the negative expression regulation (by antiregulated lncRNA) of genes with roles in processes such as the mitotic exit network ( $A M N 1, D B F 2$, and NUD1), bud site selection (GIC1, RSR1, and RAX1), cytokinesis (AIM44), mitotic transitions and checkpoints (SWE1, SPC25, and HSL1), Ras signalling (IRA1 and $B M H 2$ ), glucose signalling ( $Y C K 1$ and $Y A K 1)$, and mating signalling (DIG2, MF(ALPHA)2, and PPQ1). 247 pairs were coregulated (198) or antiregulated (49) in both U15/L15 and U6/L6 cell comparisons but not in the aerial-root cell comparison (8 of the antiregulated genes in this group encode ribosome subunits; the others are dispersed among many functional categories). 189 pairs were coregulated (122) or antiregulated (67) exclusively in the colony biofilm cell comparison, including antiregulated genes with roles in meiosis/sporulation and translation (7 each).

In the time point comparisons, 222, 27, and 2 lncRNA/ gene pairs, respectively, were antiregulated in the U15/U6, M15/M6, and L15/L6 comparisons. The fact that $89 \%$ of these pairs (222 of 251) were antiregulated in upper cells (U15/U6 comparison) suggests that IncRNA regulation of gene expression changes most during development of $U$ cells. This is consistent with the finding that most of the temporal gene-expression changes occur in upper cells, whereas temporal changes in lower cells and, in particular, the margin cell are moderate [17]. Genes encoding ribosome subunits, or involved in ribosome biogenesis or translation, typically appear DE together with neighbouring lncRNA, and while there is some degree of IncRNA/gene pair overlap between 6-day- and 15-day-old colonies, many genes seem to be selectively regulated in 6-day- or 15 -day-old colonies. The translation initiation factor gene TIF1 is upregulated, while its lncRNA is downregulated in $\mathrm{U}$ and $\mathrm{M}$ relative to $\mathrm{L}$ cells in 15-day-old colonies only, suggesting that differentiation may require divergence in fine-tuning of translation rates as colonies age. While RPL36B is upregulated (and its lncRNA downregulated) in $U$ and $M$ cells, relative to $L$ cells, of both 6-day- and 15-day-old colonies, repression of its paralog $R P L 36 A$ is relieved (potentially by lncRNA downregulation) only in 15-day-old colonies. Since deletion of the latter decreases fermentative growth but increases respiratory growth, its increased expression as the colony ages is consistent with the utilization by $\mathrm{U}$ cells of $\mathrm{L}$ cell-derived hexoses in differentiated colonies [72]. Stoichiometric changes in the ribosome subunit make-up may thus represent one aspect of the metabolic remodelling program as cells in ageing colonies differentiate.

Cell cycle progression is regulated under stress conditions by antiregulated noncoding RNAs [73] for genes such as FAR1 (encoding an inhibitor of cyclin-dependent kinase (CDK) Cdc28p involved in cell cycle phase transitions). In addition, Lardenois et al. [28] suggested that expression of the CLN2 gene that encodes cyclin G1 may be negatively regulated by promoter-overlapping MUT1465 expression, relieving CLN2-dependent repression of IME1 and allowing meiosis to proceed. In colony biofilms, we observed FAR1 expression to be upregulated 2.8-fold in aerial cells, and its antisense SUT locus SUT204 was upregulated 1.7-fold in roots. Thus, the Far1p CDK inhibitor may elicit mitotic cell cycle arrest in aerial cells, whereas its expression is repressed in roots to permit cell cycle progression. Furthermore, the cyclin CLN3 is upregulated 1.7-fold in roots and its antisense-overlapping partner MUT30.1 is upregulated 2.1fold in aerial cells. Similarly, CLN2 is upregulated 1.7-fold in roots, and the expression of MUT1465.2, which is located upstream (i.e., over the presumed promoter region) of CLN2, is upregulated 3.3-fold in aerial cells. These findings are consistent with previous findings that aerial cells have entered the stationary phase in 40-hour-old colonies, whereas root cells continue to divide [18], indicating that lncRNAs may participate in the regulation of cell division in biofilm colony cell subpopulations. The situation in smooth colonies is less clear because MUT30.1 and MUT1465.2 were not detected in smooth colonies and SUT204 is upregulated 3-fold in L relative to $U$ cells and FAR1 2.6-fold in $U$ relative to $L$ cells only in 6-day-old colonies, despite the fact that some dividing cells are present in the very upper layers of these colonies.

\section{Conclusions}

The complement of lncRNA classes (MUTs, CUTs, etc.) is cell-type-specific, implying that lncRNA expression modulates, and/or is modulated by, cell/colony differentiation. Coregulated expression of antisense-divergent lncRNA/gene pairs appears to be largely the result of bidirectional transcription of a lncRNA and a differentially expressed gene from a common start site $[14,15,28]$. Such coregulation was the most commonly observed lncRNA/coding gene interaction seen in our study of aerial-root cells of colony biofilms and of U/L cells from smooth colonies, also during age-related differentiation. On the other hand, negative 
regulation of a coding gene by a lncRNA commonly occurs in the antisense-overlapping orientation. Potential negative regulation of gene expression by antisense-overlapping lncRNAs was most commonly seen in differentiated cell subpopulations, that is, upper and lower cells of 6- and 15-day-old smooth colonies, and changes most over time (between 6 and 15 days) in upper cells. Some potential negative regulations in upper versus lower cells are common to 6-day- and 15-day-old smooth colonies, but few are shared by smooth colonies and colony biofilms, which is consistent with the different lifestyles of these two types of colony populations. Fundamental processes targeted by lncRNA-negative regulation have well-established roles in ageing and differentiation, such as meiosis/sporulation, the cell cycle, cell signalling, ribosome biogenesis, translation, and cell wall assembly/maintenance. Negative regulation of these processes by lncRNAs can enable their fine-tuning during development of yeast smooth colonies and colony biofilms. Further research will be needed to prove and clarify the role of particular lncRNAs in the differentiation of cells within aging multicellular yeast populations.

\section{Conflicts of Interest}

The authors declare that they have no conflicts of interest.

\section{Acknowledgments}

The research leading to these results has received funding from the Norwegian Financial Mechanism 2009-2014 under Project Contract no. MSMT-28477/2014 (7F14083) and Czech Science Foundation 13-08605S. Derek Wilkinson and Zdena Palková are also supported by LQ1604 NPU II provided by MEYS and Libuše Váchová by RVO61388971, and the research was performed in BIOCEV supported by CZ.1.05/1.1.00/02.0109 BIOCEV provided by ERDF and MEYS.

\section{References}

[1] Z. Palkova and L. Vachova, "Life within a community: benefit to yeast long-term survival," FEMS Microbiology Reviews, vol. 30, no. 5, pp. 806-824, 2006.

[2] S. Brückner and H. U. Mösch, "Choosing the right lifestyle: adhesion and development in Saccharomyces cerevisiae," FEMS Microbiology Reviews, vol. 36, no. 1, pp. 25-58, 2012.

[3] S. M. Honigberg, "Cell signals, cell contacts, and the organization of yeast communities," Eukaryotic Cell, vol. 10, no. 4, pp. 466-473, 2011.

[4] Z. Palkova and L. Vachova, "Yeast cell differentiation: lessons from pathogenic and non-pathogenic yeasts," Seminars in Cell \& Developmental Biology, vol. 57, pp. 110-119, 2016.

[5] Z. Palkova, D. Wilkinson, and L. Vachova, "Aging and differentiation in yeast populations: elders with different properties and functions," FEMS Yeast Research, vol. 14, no. 1, pp. 96108, 2014.

[6] K. Podholová, V. Plocek, S. Rešetárová et al., "Divergent branches of mitochondrial signaling regulate specific genes and the viability of specialized cell types of differentiated yeast colonies," Oncotarget, vol. 7, no. 13, pp. 15299-15314, 2016.
[7] Z. Tan, M. Hays, G. A. Cromie et al., "Aneuploidy underlies a multicellular phenotypic switch," Proceedings of the National Academy of Sciences of the United States of America, vol. 110, no. 30, pp. 12367-12372, 2013.

[8] A. Fatica and I. Bozzoni, "Long non-coding RNAs: new players in cell differentiation and development," Nature Reviews Genetics, vol. 15, no. 1, pp. 7-21, 2014.

[9] A. M. Burroughs, Y. Ando, and L. Aravind, "New perspectives on the diversification of the RNA interference system: insights from comparative genomics and small RNA sequencing," Wiley Interdisciplinary Reviews: RNA, vol. 5, no. 2, pp. 141-181, 2014.

[10] L. David, W. Huber, M. Granovskaia et al., "A high-resolution map of transcription in the yeast genome," Proceedings of the National Academy of Sciences of the United States of America, vol. 103, no. 14, pp. 5320-5325, 2006.

[11] C. A. Davis and M. Ares, "Accumulation of unstable promoter-associated transcripts upon loss of the nuclear exosome subunit Rrp6p in Saccharomyces cerevisiae," Proceedings of the National Academy of Sciences of the United States of America, vol. 103, no. 9, pp. 3262-3267, 2006.

[12] F. Wyers, M. Rougemaille, G. Badis et al., "Cryptic pol II transcripts are degraded by a nuclear quality control pathway involving a new poly(A) polymerase," Cell, vol. 121, no. 5, pp. 725-737, 2005.

[13] E. A. Alcid and T. Tsukiyama, "Expansion of antisense lncRNA transcriptomes in budding yeast species since the loss of RNAi," Nature Structural \& Molecular Biology, vol. 23, no. 5, pp. 450-455, 2016.

[14] H. Neil, C. Malabat, Y. d'Aubenton-Carafa, Z. Xu, L. M. Steinmetz, and A. Jacquier, "Widespread bidirectional promoters are the major source of cryptic transcripts in yeast," Nature, vol. 457, no. 7232, pp. 1038-1042, 2009.

[15] Z. Xu, W. Wei, J. Gagneur et al., "Bidirectional promoters generate pervasive transcription in yeast," Nature, vol. 457, no. 7232, pp. 1033-1037, 2009.

[16] J. Maršíková, D. Wilkinson, O. Hlaváček et al., "Metabolic differentiation of surface and invasive cells of yeast colony biofilms revealed by gene expression profiling," BMC Genomics, vol. 18 , no. 1, p. 814, 2017.

[17] D. Wilkinson, J. Maršíková, O. Hlaváček et al., “Transcriptome remodeling of differentiated cells during chronological ageing of yeast colonies: new insights into metabolic differentiation," Oxidative Medicine and Cellular Longevity, vol. 2018, Article ID 4932905, 17 pages, 2018.

[18] L. Váchová, V. Stovícek, O. Hlavácek et al., "Flo11p, drug efflux pumps, and the extracellular matrix cooperate to form biofilm yeast colonies," The Journal of Cell Biology, vol. 194, no. 5, pp. 679-687, 2011.

[19] M. Cap, L. Stepanek, K. Harant, L. Vachova, and Z. Palkova, "Cell differentiation within a yeast colony: metabolic and regulatory parallels with a tumor-affected organism," Molecular Cell, vol. 46, no. 4, pp. 436-448, 2012.

[20] F. F. Costa, "Non-coding RNAs: meet thy masters," BioEssays, vol. 32, no. 7, pp. 599-608, 2010.

[21] T. N. Mavrich, I. P. Ioshikhes, B. J. Venters et al., "A barrier nucleosome model for statistical positioning of nucleosomes throughout the yeast genome," Genome Research, vol. 18, no. 7, pp. 1073-1083, 2008.

[22] D. Schulz, B. Schwalb, A. Kiesel et al., "Transcriptome surveillance by selective termination of noncoding RNA synthesis," Cell, vol. 155, no. 5, pp. 1075-1087, 2013. 
[23] J. Wu, D. Delneri, and R. T. O'Keefe, "Non-coding RNAs in Saccharomyces cerevisiae: what is the function?," Biochemical Society Transactions, vol. 40, no. 4, pp. 907-911, 2012.

[24] M. Tisseur, M. Kwapisz, and A. Morillon, "Pervasive transcription-lessons from yeast," Biochimie, vol. 93, no. 11, pp. 1889-1896, 2011.

[25] J. M. Vera and R. D. Dowell, "Survey of cryptic unstable transcripts in yeast," BMC Genomics, vol. 17, no. 1, p. 305, 2016.

[26] M. Yassour, J. Pfiffner, J. Z. Levin et al., "Strand-specific RNA sequencing reveals extensive regulated long antisense transcripts that are conserved across yeast species," Genome Biology, vol. 11, no. 8, article R87, 2010.

[27] B. Gelfand, J. Mead, A. Bruning et al., "Regulated antisense transcription controls expression of cell-type-specific genes in yeast," Molecular and Cellular Biology, vol. 31, no. 8, pp. 1701-1709, 2011.

[28] A. Lardenois, Y. Liu, T. Walther et al., "Execution of the meiotic noncoding RNA expression program and the onset of gametogenesis in yeast require the conserved exosome subunit Rrp6," Proceedings of the National Academy of Sciences of the United States of America, vol. 108, no. 3, pp. 1058-1063, 2011.

[29] F. J. van Werven, G. Neuert, N. Hendrick et al., "Transcription of two long noncoding RNAs mediates mating-type control of gametogenesis in budding yeast," Cell, vol. 150, no. 6, pp. 1170-1181, 2012.

[30] T. Kim, Z. Xu, S. Clauder-Munster, L. M. Steinmetz, and S. Buratowski, "Set3 HDAC mediates effects of overlapping noncoding transcription on gene induction kinetics," Cell, vol. 150, no. 6, pp. 1158-1169, 2012.

[31] I. Toesca, C. R. Nery, C. F. Fernandez, S. Sayani, and G. F. Chanfreau, "Cryptic transcription mediates repression of subtelomeric metal homeostasis genes," PLoS Genetics, vol. 7, no. 6, article e1002163, 2011.

[32] M. Nadal-Ribelles, C. Solé, Z. Xu, L. M. Steinmetz, E. de Nadal, and F. Posas, "Control of Cdc28 CDK1 by a stress-induced lncRNA,” Molecular Cell, vol. 53, no. 4, pp. 549-561, 2014.

[33] K. Vaškovičová, T. Awadová, P. Veselá, M. Balážová, M. Opekarová, and J. Malinsky, "mRNA decay is regulated via sequestration of the conserved $5^{\prime}-3^{\prime}$ exoribonuclease Xrn1 at eisosome in yeast," European Journal of Cell Biology, vol. 96, no. 6, pp. 591-599, 2017.

[34] E. L. van Dijk, C. L. Chen, Y. d'Aubenton-Carafa et al., "XUTs are a class of Xrn1-sensitive antisense regulatory non-coding RNA in yeast," Nature, vol. 475, no. 7354, pp. 114-117, 2011.

[35] B. Luke, A. Panza, S. Redon, N. Iglesias, Z. Li, and J. Lingner, “The Rat1p 5' to 3 ' exonuclease degrades telomeric repeatcontaining RNA and promotes telomere elongation in Saccharomyces cerevisiae," Molecular Cell, vol. 32, no. 4, pp. 465-477, 2008.

[36] S. A. Cakiroglu, J. B. Zaugg, and N. M. Luscombe, "Backmasking in the yeast genome: encoding overlapping information for protein-coding and RNA degradation," Nucleic Acids Research, vol. 44, no. 17, pp. 8065-8072, 2016.

[37] J. T. Arigo, D. E. Eyler, K. L. Carroll, and J. L. Corden, "Termination of cryptic unstable transcripts is directed by yeast RNA-binding proteins Nrd1 and Nab3," Molecular Cell, vol. 23, no. 6, pp. 841-851, 2006.

[38] M. M. Darby, L. Serebreni, X. Pan, J. D. Boeke, and J. L. Corden, "The Saccharomyces cerevisiae Nrd1-Nab3 transcription termination pathway acts in opposition to Ras signaling and mediates response to nutrient depletion," Molecular and Cellular Biology, vol. 32, no. 10, pp. 1762-1775, 2012.

[39] J. Houseley, "Form and function of eukaryotic unstable noncoding RNAs," Biochemical Society Transactions, vol. 40, no. 4, pp. 836-841, 2012.

[40] M. Wery, M. Descrimes, N. Vogt, A. S. Dallongeville, D. Gautheret, and A. Morillon, "Nonsense-mediated decay restricts LncRNA levels in yeast unless blocked by doublestranded RNA structure," Molecular Cell, vol. 61, no. 3, pp. 379-392, 2016.

[41] S. L. Bumgarner, R. D. Dowell, P. Grisafi, D. K. Gifford, and G. R. Fink, "Toggle involving cis-interfering noncoding RNAs controls variegated gene expression in yeast," Proceedings of the National Academy of Sciences of the United States of America, vol. 106, no. 43, pp. 18321-18326, 2009.

[42] Y. Mostovoy, A. Thiemicke, T. Y. Hsu, and R. B. Brem, "The role of transcription factors at antisense-expressing gene pairs in yeast," Genome Biology and Evolution, vol. 8, no. 6, pp. 1748-1761, 2016.

[43] S. L. Bumgarner, G. Neuert, B. F. Voight et al., "Single-cell analysis reveals that noncoding RNAs contribute to clonal heterogeneity by modulating transcription factor recruitment," Molecular Cell, vol. 45, no. 4, pp. 470-482, 2012.

[44] A. Cassone and R. Cauda, "Candida and candidiasis in HIV-infected patients: where commensalism, opportunistic behavior and frank pathogenicity lose their borders," AIDS, vol. 26, no. 12, pp. 1457-1472, 2012.

[45] C. J. Nobile and A. P. Mitchell, "Regulation of cell-surface genes and biofilm formation by the C. albicans transcription factor Bcrlp," Current Biology, vol. 15, no. 12, pp. 11501155, 2005.

[46] G. Ramage, R. Rajendran, L. Sherry, and C. Williams, "Fungal biofilm resistance," International Journal of Microbiology, vol. 2012, Article ID 528521, 14 pages, 2012.

[47] J. A. Martens, L. Laprade, and F. Winston, "Intergenic transcription is required to repress the Saccharomyces cerevisiae SER3 gene," Nature, vol. 429, no. 6991, pp. 571-574, 2004.

[48] J. A. Martens, P. Y. Wu, and F. Winston, "Regulation of an intergenic transcript controls adjacent gene transcription in Saccharomyces cerevisiae," Genes \& Development, vol. 19, no. 22, pp. 2695-2704, 2005.

[49] E. M. Prescott and N. J. Proudfoot, "Transcriptional collision between convergent genes in budding yeast," Proceedings of the National Academy of Sciences of the United States of America, vol. 99, no. 13, pp. 8796-8801, 2002.

[50] M. Pinskaya, S. Gourvennec, and A. Morillon, "H3 lysine 4 di- and tri-methylation deposited by cryptic transcription attenuates promoter activation," The EMBO Journal, vol. 28, no. 12, pp. 1697-1707, 2009.

[51] S. Geisler, L. Lojek, A. M. Khalil, K. E. Baker, and J. Coller, "Decapping of long noncoding RNAs regulates inducible genes," Molecular Cell, vol. 45, no. 3, pp. 279-291, 2012.

[52] T. L. Lenstra, A. Coulon, C. C. Chow, and D. R. Larson, "Single-molecule imaging reveals a switch between spurious and functional ncRNA transcription," Molecular Cell, vol. 60, no. 4, pp. 597-610, 2015.

[53] Z. T. Beck, S. C. Cloutier, M. J. Schipma et al., "Regulation of glucose-dependent gene expression by the RNA helicase Dbp2 in Saccharomyces cerevisiae," Genetics, vol. 198, no. 3, pp. 1001-1014, 2014. 
[54] S. C. Cloutier, W. K. Ma, L. T. Nguyen, and E. J. Tran, “The DEAD-box RNA helicase Dbp2 connects RNA quality control with repression of aberrant transcription," The Journal of Biological Chemistry, vol. 287, no. 31, pp. 26155-26166, 2012.

[55] S. C. Cloutier, S. Wang, W. K. Ma et al., "Regulated formation of lncRNA-DNA hybrids enables faster transcriptional induction and environmental adaptation," Molecular Cell, vol. 61, no. 3, pp. 393-404, 2016.

[56] S. C. Cloutier, S. Wang, W. K. Ma, C. J. Petell, and E. J. Tran, "Long noncoding RNAs promote transcriptional poising of inducible genes," PLoS Biology, vol. 11, no. 11, article e1001715, 2013.

[57] I. Zacharioudakis and D. Tzamarias, "Bimodal expression of yeast GAL genes is controlled by a long non-coding RNA and a bifunctional galactokinase," Biochemical and Biophysical Research Communications, vol. 486, no. 1, pp. 63-69, 2017.

[58] J. Berretta, M. Pinskaya, and A. Morillon, "A cryptic unstable transcript mediates transcriptional trans-silencing of the Ty1 retrotransposon in S. cerevisiae," Genes \& Development, vol. 22, no. 5, pp. 615-626, 2008.

[59] J. Camblong, N. Beyrouthy, E. Guffanti, G. Schlaepfer, L. M. Steinmetz, and F. Stutz, "Trans-acting antisense RNAs mediate transcriptional gene cosuppression in S. cerevisiae," Genes \& Development, vol. 23, no. 13, pp. 1534-1545, 2009.

[60] J. P. Uhler, C. Hertel, and J. Q. Svejstrup, "A role for noncoding transcription in activation of the yeast PHO5 gene," Proceedings of the National Academy of Sciences of the United States of America, vol. 104, no. 19, pp. 8011-8016, 2007.

[61] D. Bunina, M. Štefl, F. Huber et al., "Upregulation of SPS100 gene expression by an antisense RNA via a switch of mRNA isoforms with different stabilities," Nucleic Acids Research, vol. 45, no. 19, pp. 11144-11158, 2017.

[62] M. Chia, A. Tresenrider, J. Chen, G. Spedale, V. Jorgensen et al., "Transcription of a 5 ' extended mRNA isoform directs dynamic chromatin changes and interference of a downstream promoter," eLife, vol. 6, article 27420, 2017.

[63] J. H. Kim, B. B. Lee, Y. M. Oh et al., "Modulation of mRNA and lncRNA expression dynamics by the Set2-Rpd3S pathway," Nature Communications, vol. 7, article 13534, 2016.

[64] S. L. McDaniel, A. J. Hepperla, J. Huang et al., "H3K36 methylation regulates nutrient stress response in Saccharomyces cerevisiae by enforcing transcriptional fidelity," Cell Reports, vol. 19, no. 11, pp. 2371-2382, 2017.

[65] M. Kwapisz, M. Ruault, E. van Dijk et al., "Expression of subtelomeric lncRNAs links telomeres dynamics to RNA decay in S. cerevisiae," Non-Coding RNA, vol. 1, no. 2, pp. 94-126, 2015.

[66] A. Traven, A. Jänicke, P. Harrison, A. Swaminathan, T. Seemann, and T. H. Beilharz, "Transcriptional profiling of a yeast colony provides new insight into the heterogeneity of multicellular fungal communities," PLoS One, vol. 7, no. 9, article e46243, 2012.

[67] L. Vachova, L. Hatakova, M. Cap, M. Pokorna, and Z. Palkova, "Rapidly developing yeast microcolonies differentiate in a similar way to aging giant colonies," Oxidative Medicine and Cellular Longevity, vol. 2013, Article ID 102485, 9 pages, 2013.

[68] S. San Paolo, S. Vanacova, L. Schenk et al., "Distinct roles of non-canonical poly(A) polymerases in RNA metabolism," PLoS Genetics, vol. 5, no. 7, article e1000555, 2009.

[69] J. Houseley and D. Tollervey, "Yeast Trf5p is a nuclear poly(A) polymerase," EMBO Reports, vol. 7, no. 2, pp. 205-211, 2006.
[70] J. M. Cherry, “The Saccharomyces genome database: advanced searching methods and data mining," Cold Spring Harb Protoc, vol. 2015, no. 12, article pdb.prot088906, 2015.

[71] A. Khan and A. Mathelier, "Intervene: a tool for intersection and visualization of multiple gene or genomic region sets," BMC Bioinformatics, vol. 18, no. 1, p. 287, 2017.

[72] M. Cap, L. Vachova, and Z. Palkova, "Longevity of U cells of differentiated yeast colonies grown on respiratory medium depends on active glycolysis," Cell Cycle, vol. 14, no. 21, pp. 3488-3497, 2015.

[73] C. Sole, M. Nadal-Ribelles, E. de Nadal, and F. Posas, "A novel role for lncRNAs in cell cycle control during stress adaptation," Current Genetics, vol. 61, no. 3, pp. 299-308, 2015. 


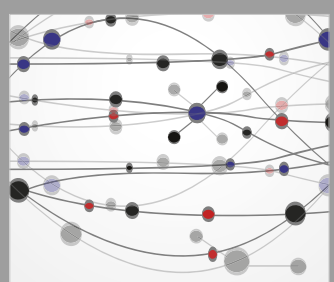

The Scientific World Journal
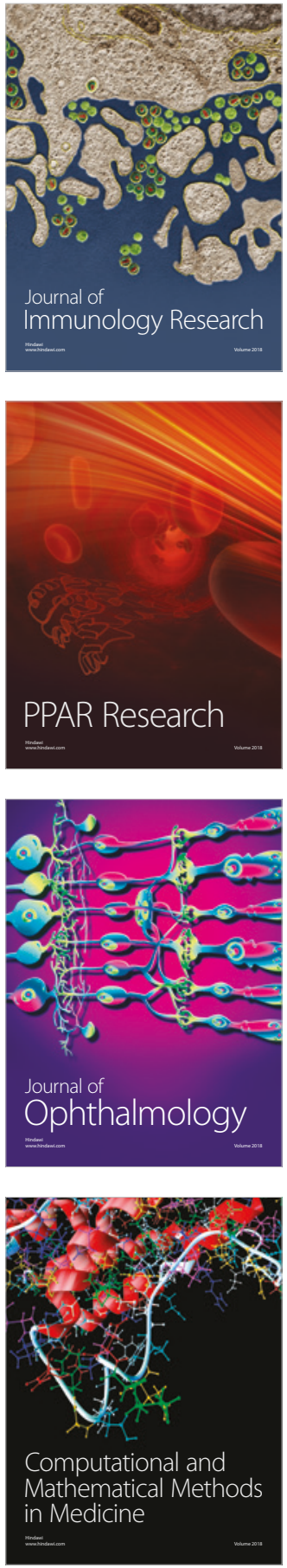

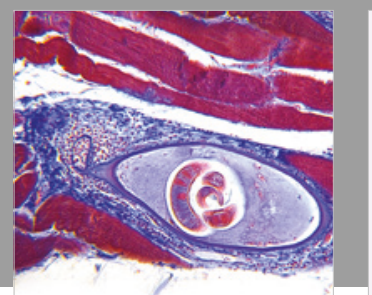

Gastroenterology Research and Practice

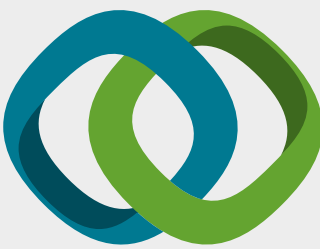

\section{Hindawi}

Submit your manuscripts at

www.hindawi.com
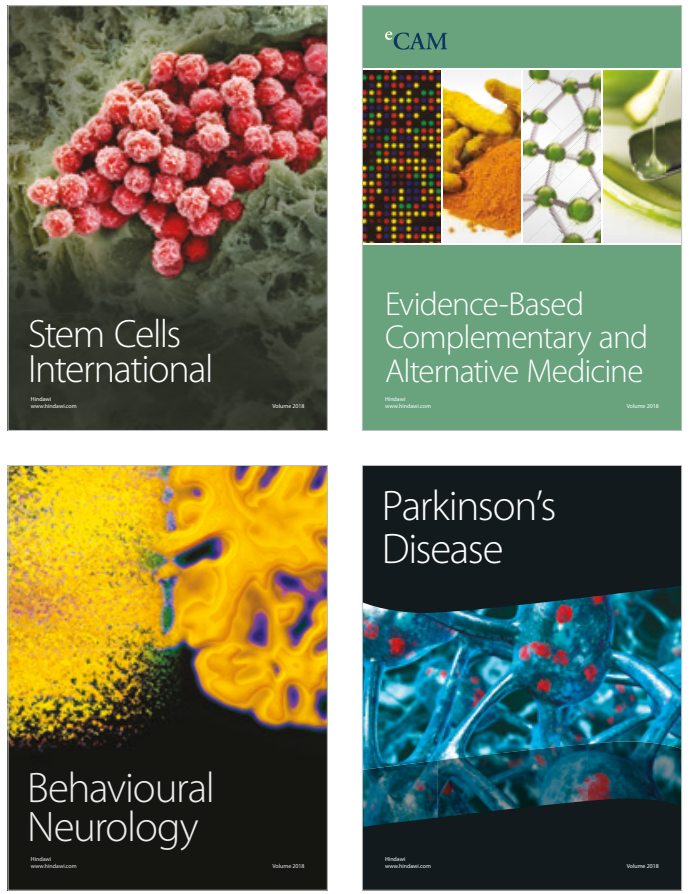

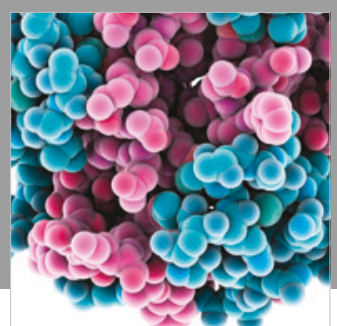

ournal of

Diabetes Research

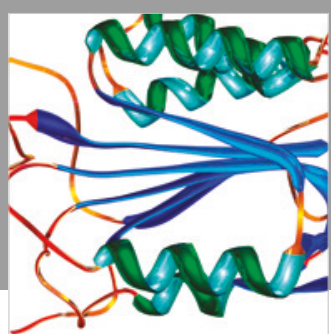

Disease Markers
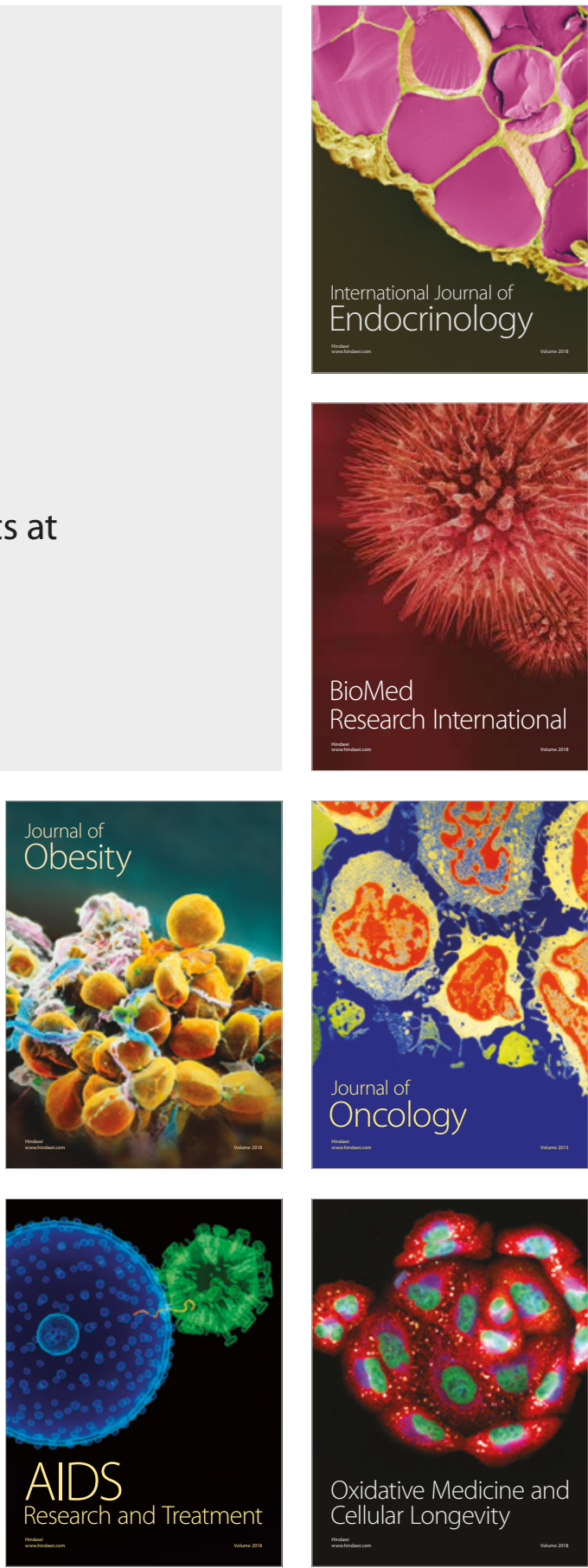